\title{
Electric field phase diagram of thiourea determined by optical birefringence
}

\author{
J. P. Jamet \\ Laboratoire de Physique des Solides (*), Université Paris-Sud, Bâtiment 510, 91405 Orsay, France.
}

(Reçu le 18 novembre 1980, accepté le 30 janvier 1981)

\begin{abstract}
Résumé. - Le diagramme de phase de la thiourée $\mathrm{SC}\left(\mathrm{ND}_{2}\right)_{2}$ dans le plan $(E, T)$ a été déterminé par des mesures de biréfringence linéaire. A notre connaissance, c'est la première fois qu'un tel diagramme est déterminé dans un système qui présente des transitions vers une phase incommensurable. La ligne de transition se divise en trois parties : une ligne de premier ordre $T_{\mathrm{H}}(E)$, séparant les phases ferroélectrique et incommensurable, qui se termine à un champ maximum $E_{\mathrm{M}}=2285 \pm 10 \mathrm{~V} / \mathrm{mm}$ avec $T_{\mathrm{M}}=210 \pm 0,2 \mathrm{~K}$ où le saut de biréfringence s'annule; une ligne de second ordre $T_{\lambda}(E)$ séparant les phases paraélectrique et incommensurable, qui se termine à un point $E_{\mathrm{tr}} \simeq 2050 \mathrm{~V} / \mathrm{mm}, T_{\mathrm{tr}} \simeq 212,5 \mathrm{~K}$; entre $\left(E_{\mathrm{M}}, T_{\mathrm{M}}\right)$ et $\left(E_{\mathrm{tr}}, T_{\mathrm{tr}}\right)$, la ligne de transition paraît être faiblement de premier ordre.
\end{abstract}

\begin{abstract}
The phase diagram of thiourea : $\mathrm{SC}\left(\mathrm{ND}_{2}\right)_{2}$ in the $(E, T)$ plane has been determined by linear birefringence measurements. To our knowledge, it is the first time that such diagram has been determined in a system which undergoes transitions to an incommensurate phase. The transition line consists of three parts : a first-order line $T_{\mathrm{H}}(E)$ separating the ferroelectric phase from the incommensurate phase which terminates at a maximum field $E_{\mathrm{M}}=2285 \pm 10 \mathrm{~V} / \mathrm{mm}$ with $T_{\mathrm{M}}=210 \pm 0.2 \mathrm{~K}$, where the birefringence jump is zero, a second-order line $T_{\lambda}(E)$ separating the paraelectric phase from the incommensurate one which terminates at a point
\end{abstract}

$$
E_{\mathrm{tr}} \simeq 2050 \mathrm{~V} / \mathrm{mm}, \quad T_{\mathrm{tr}} \simeq 212.5 \mathrm{~K} ;
$$

between $\left(E_{\mathrm{M}}, T_{\mathrm{M}}\right)$ and $\left(E_{\mathrm{tr}}, T_{\mathrm{tr}}\right)$ the transition line looks to be weakly first-order.

1. Introduction. - Thiourea $\left(\mathrm{SC}\left(\mathrm{ND}_{2}\right)_{2}\right)$ has been the subject of many experimental, as well as theoretical investigations over the last few years, due essentially to the presence of an incommensurate phase. Besides the fundamental neutron and X-ray studies, the $(P, T)$ diagram has been measured very recently [1] by neutron diffraction studies. Some preliminary results are presented here concerning the $(\mathbf{E}, T)$ diagram, where $\mathbf{E}$ is an applied d.c. electric field, by measuring the optical linear birefringence; some preliminary measurements with zero electric field made with the same technique have already been published [2].

At atmospheric pressure and zero electric field, the following phases of $\mathrm{SC}\left(\mathrm{ND}_{2}\right)_{2}$ are found successively with increasing temperature : orthorhombic ferroelectric, with a net dipolar moment along the a-axis, below $T_{\mathrm{H}}=190 \mathrm{~K}$; commensurate with a $9 \mathrm{~b}$ superstructure up to $192 \mathrm{~K}$; then incommensurate; and above $T_{\lambda}=216 \mathrm{~K}$ thiourea is paraelectric. At $T_{\mathrm{H}}$ the transition is first-order and at $T_{\lambda}$

(*) Associé au C.N.R.S. it is second-order. We have shown previously [2] that in the incommensurate phase, the birefringence changes are proportional to the square of the order parameter in zero field, i.e., $\Delta n_{b c}=A\left\langle\varphi_{b}^{2}\right\rangle$ (if one neglects fluctuations) [3] where $\varphi_{b}$ represents the mean rotational and translational motion of the thiourea molecule around the b-axis, and $A$ is a function of the fourth-order photoelastic tensor components. In zero $E$-field we have found that : $\varphi_{b} \sim\left(T-T_{\lambda}\right)^{0.38 \pm 0.01}$.

2. Electric field phase diagram. - We briefly recall the technique we have used to measure the birefringence. A photoelastic modulator switches the polarization of a laser beam $(6328 \AA)$ along two perpendicular directions at $50 \mathrm{kHz}$; these directions are in the b, c crystallographic plane of the sample. The optical signal is compensated, decomposed by an analyser and detected by a photomultiplier. A lock-in amplifier is used to measure the electrical signal given by the photomultiplier. For the usual case of large birefringence variations, we made use of a calibrated compensator to cancel the detected signal. Very 
small birefringence variations can be measured : typically $\delta(\Delta n) \sim 10^{-8}$. The cleaved samples of $\mathrm{SC}\left(\mathrm{ND}_{2}\right)_{2}$ (typically : $0.7 \mathrm{~mm}$ thick) were gold-plated under vacuum on two opposite $a$-faces where $\mathbf{a}$ is the ferroelectric axis and is also parallel to the laser beam in our experimental configuration. The gold thickness was determined so that the laser beam could penetrate it (over all optical density of the order of unity).

The crystals were glued onto the cold finger of a gas-flow cryostat and the temperature was measured with a Lake Shore calibrated Si-diode thermometer glued as close as possible to the sample. A diaphragm $(0.1 \mathrm{~mm}$ inner diameter) was placed at the centre of the sample so as to probe only its central part and to avoid as much as possible the influence of the electrical gradient at the edges.

The measurements presented here were made at fixed voltages by varying the temperature at rates of less than $1 \mathrm{mK} / \mathrm{s}$ to ensure good thermal homogeneity.

3. Experimental results. - The $b c$-plane linear birefringence in the incommensurate phase is presented in figures 1 and 2 as a function of temperature for various electrical fields; the zero of the birefringence is arbitrary but is the same in both figures. The thickness of the curves represents the accuracy of the experimental determination. All plots presented here were made with decreasing temperature, but because of difficulties, particularly with electrical breakdown, they were obtained on three different samples and renormalized with respect to one another. Some spread of the first-order transitions at $E \neq 0$ was observed which is probably due to electrical field inhomogeneity inside the sample.

As can clearly be seen, increasing $E$ leads to an increase of the first-order transition temperature $T_{\mathrm{H}}(E)$; the rather large, rate of increase of $T_{\mathrm{H}}$ with $E$ is due to the polar character of the ferroelectric phase. On the contrary, the second-order transi-

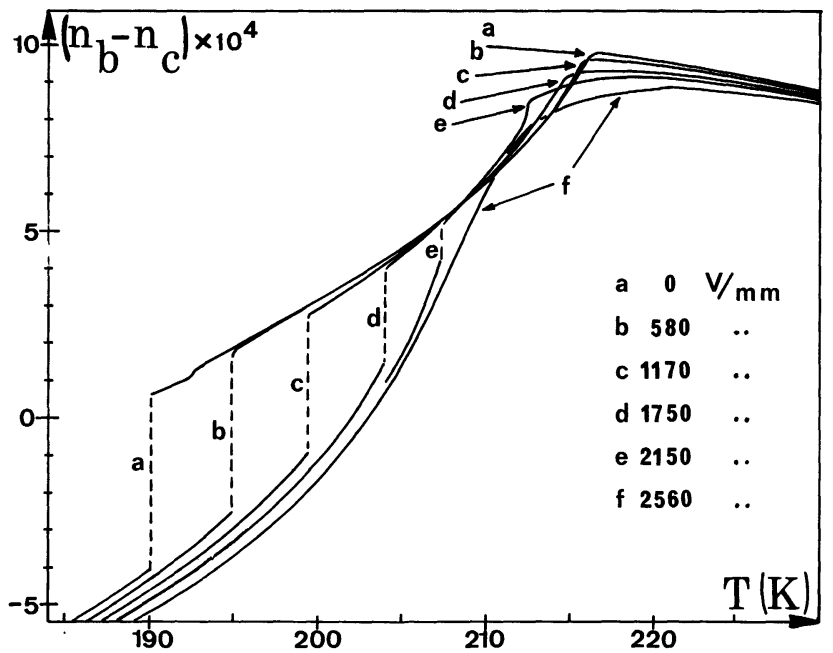

Fig. 1. - Experimental birefringence cooling curves in an extended $E$ range. The lock-in at $q_{0}=b^{*} / 9$ is visible on curve « a " at about $192 \mathrm{~K}$. The zero of birefringence is arbitrary.

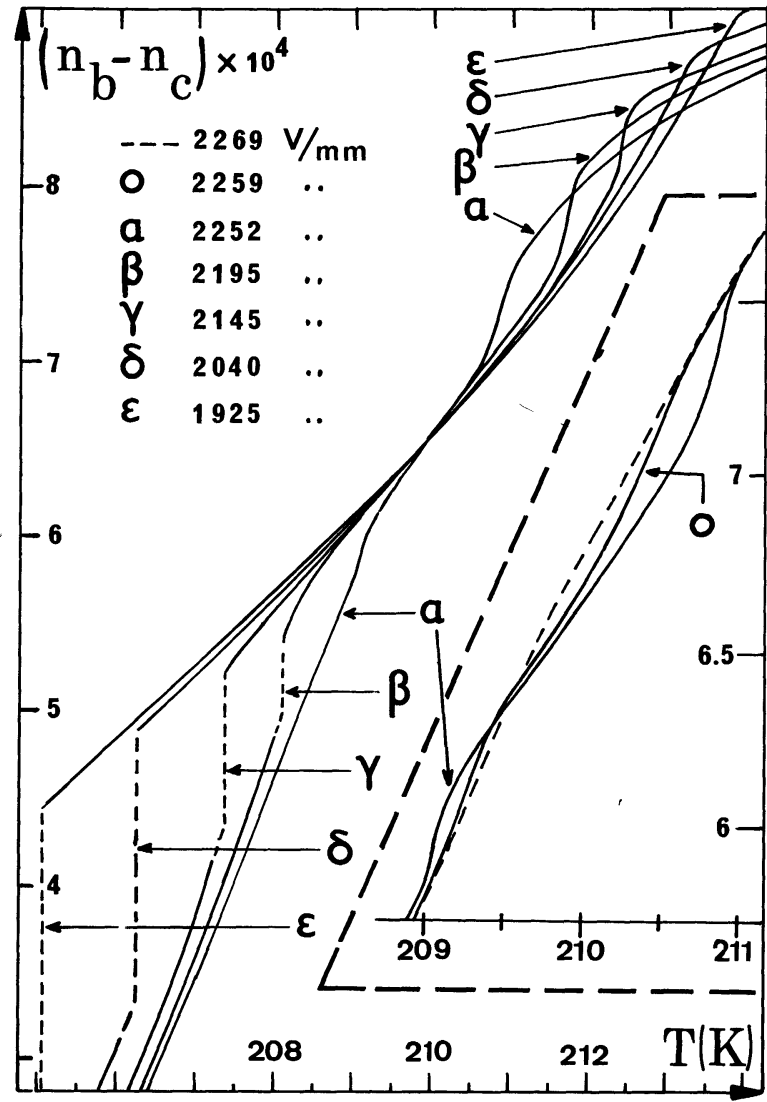

Fig. 2. - Experimental birefringence cooling curves for $E$-values around the $\left(E_{\mathrm{M}}, T_{\mathrm{M}}\right)$ point (see text). The insert shows the experimental curves on an extended scale. The crossing of all the curves seen at around $210 \mathrm{~K}$ (for $E<E_{\mathrm{M}}$ ) is a feature of the experiment that we do not understand. As for figure 1, these curves are not corrected for the variation of the crystallographic parameter " $a$ " along which the light beam propagates.

tion temperature $T_{\lambda}(E)$ is relatively independent of $E$ at low values of $E$, which is related to the nonpolar character of the incommensurate phase. Simultaneously the first-order birefringence jump is progressively reduced as $E$ increases, and the diffusion by domains at $T_{\mathrm{H}}(E)$ which is very strong in zero field, vanishes in high fields.

4. Phase diagram $(E, T)$. - The phase diagram $(E, T)$ is shown in figure 3. The black triangles give the positions of the first-order transitions $T_{\mathrm{H}}(E)$ with decreasing $T$ and the clear triangles with increasing $T$. The slope of the line which separates the ferroelectric zone II from the incommensurate zone III is constant up to about $2 \mathrm{kV} / \mathrm{mm}$ beyond which it begins to saturate. (Indeed on the dotted curve of figure 2 corresponding to $2269 \mathrm{~V} / \mathrm{mm}$, the firstorder transition is still just visible.) The slope of the linear part of the first-order curve is $8 \mathrm{mK} / \mathrm{V} / \mathrm{mm}$. The birefringence jump at the transition decreases with increasing $E$, becoming zero at the point

$$
E_{\mathrm{M}}=2285 \pm 10 \mathrm{~V} / \mathrm{mm}, \quad T_{\mathrm{M}}=210 \pm 0.2 \mathrm{~K},
$$

where the slope of the curve is zero. 


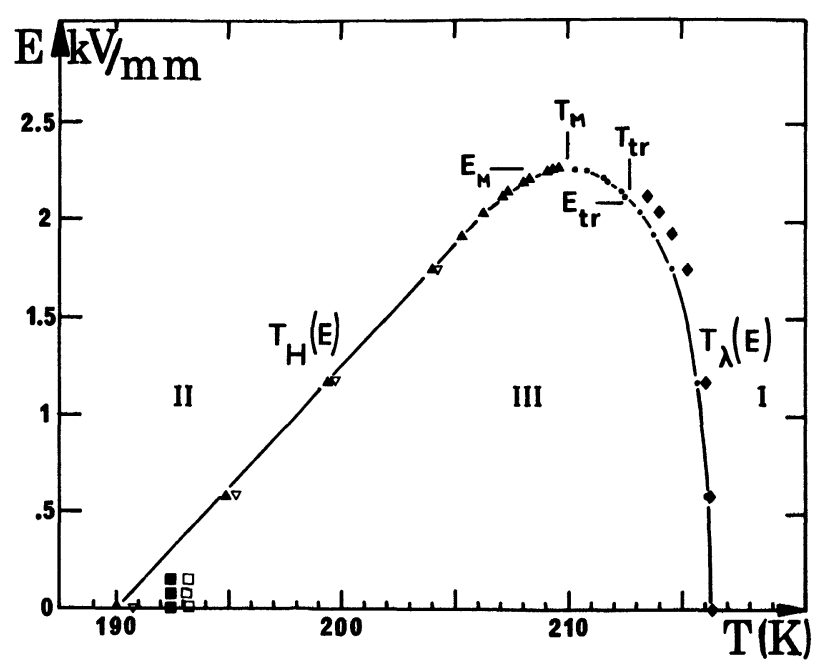

Fig. 3. $-E, T$ diagram. The lines are a guide to the eye. For meaning of symbols, see text.

On the other hand the black diamonds in figure 3 represent the second-order transition temperature $T_{\lambda}(E)$ as determined by a power law fit with the same base line as in [2] $(E=0)$. However the uncertainty, due to the decreasing extent of the incommensurate phase as $E$ increases, makes this determination less and less accurate, so we have also reported (black dots) the temperatures of the inflexion points on the experimental curves of figures 1 and 2 which can be taken as the second-order phase transition temperatures. The second-order transition curve $T_{\lambda}(E)$ which separates the incommensurate phase II from paraelectric phase I begins with an infinite slope at low $E$-values; nevertheless a careful examination of the experimental curves (see for example " $\mathrm{e}$ " in figure 1 or " $\gamma$ » in figure 2) seems to show that the transition is weakly first-order for field values larger than about $E_{\mathrm{tr}}=2050 \mathrm{~V} / \mathrm{mm}\left(T_{\mathrm{tr}} \simeq 212.5 \mathrm{~K}\right)$ and up to $\left(E_{\mathrm{M}}, T_{\mathrm{M}}\right)$. Between the critical point $\left(E_{\mathrm{M}}, T_{\mathrm{M}}\right)$ and the tricritical point $\left(E_{\mathrm{tr}}, T_{\mathrm{tr}}\right)$, the birefringence jump appears to be different from zero.

The black and clear squares represent respectively the positions of the lock-in at $q_{0}=b^{*} / 9$ with decreasing and increasing temperature; as for the second-order phase transition (non-polar character), its temperature does not change with $E$ at low $E$.

5. Conclusion. - We have determined the phase diagram of deuterated thiourea in an electric field, and have found two critical points where the transitions are second-order; other experiments are being made to investigate the transition segment between the points $\left(E_{\mathrm{M}}, T_{\mathrm{M}}\right)$ and $\left(E_{\mathrm{tr}}, T_{\mathrm{tr}}\right)$ and, in particular, to determine the evolution of the birefringence jump with temperature and to fit the results with theoretical models $[4,5]$.

The birefringence cannot give the value of the incommensurate wave vector; nevertheless one can deduce from the experimental data that the absence of a birefringence jump at the point $\left(E_{\mathrm{M}}, T_{\mathrm{M}}\right)$ means that the amplitude of the incommensurability modulation is nul at this point, and that it is the same at the tricritical point $\left(E_{\mathrm{tr}}, T_{\mathrm{tr}}\right)$.

Acknowledgments. - The author would like to thank Prof. J. P. Chapelle for kindly supplying the crystals and M. Boix for gold-plating of the cleaved samples. We also acknowledge J. Ferre, F. Denoyer, A. M. Quittet, A. Moudden and S. Aubry for helpful comments and P. Lederer and Prof. C. M. Chaves for illuminating discussions.

\section{References}

[1] Denoyer, F., Moudden, A., Bellamy, A., Currat, R., VetTIER, C., LAMBERT, M., J. Physique Lett., to be published. MoudDEN, A., Thèse d'Etat, Orsay (1980), unpublished.

[2] JAMET, J. P., Quirtet, A. M., Moudden, A., E.P.S. Conference Anvers (Belgium), April 1980, to be published in Solid State Commun.
[3] Gehring, G. A., J. Phys. C : Solid State Phys. 10 (1977) 531.

[4] Michelson, A., Phys. Rev. B 16 (1977) 577.

Hornreich, R. M., Luban, M. and Shtrikman, S., Phys. Rev. Lett. 35 (1975) 1678.

[5] Lederer, P., Chaves, C. M., J. Physique Lett. 42 (1981) L-127. 